\title{
Fourier transform analysis of irradiated Weiss oscillations.
}

\author{
Jesús IÑarrea ${ }^{1,2}$ and Gloria Platero ${ }^{2}$ \\ ${ }^{1}$ Escuela Politécnica Superior, Universidad Carlos III,Leganes,Madrid,Spain and ${ }^{2}$ Unidad Asociada al Instituto de \\ Ciencia de Materiales, CSIC, Cantoblanco,Madrid,28049,Spain.
}

PACS $\mathrm{nn} \cdot \mathrm{mm} \cdot \mathrm{xx}-$ First pacs description

PACS $\mathrm{nn} . \mathrm{mm} \cdot \mathrm{xx}$ - Second pacs description

PACS $n n . m m . x x-$ Third pacs description

\begin{abstract}
We present a theoretical approach to study the effect of microwave radiation on the magnetoresistance of a one-dimensional superlattice. In our proposal the magnetoresistance of a unidirectional spatial periodic potential (superlattice), is modulated by microwave radiation due to an interference effect between both, space and time-dependent potentials. The final magnetoresistance will mainly depend on the spatial period of the superlattice and the radiation frequency. We consider an approach to study these effects based on the fast Fourier transform of the obtained magnetorresistance profiles in function of the inverse of the magnetic field. Based on this theory we propose the design of a novel radiation sensor for the Terahertz band.
\end{abstract}

Introduction. - Nowadays magnetotransport properties of highly mobile 2DES are a subject of increasing interest. In particular the study of the effects that radiation can produce on these nano-devices is attracting much attention [1]. Besides, the interplay of two different periodic modulations in these physical systems will give rise to interesting features in electron dynamics and transport. For instance, the study of the effect of microwave (MW) radiation on the transport properties of a one-dimensional superlattice in the presence of a perpendicular magnetic field, $(B)$ giving rise to Weiss oscillations [2, 4] represents a topic of great interest. Especially if we consider that MW radiation gives rise also to magnetoresistance $\left(R_{x x}\right)$ oscillations in 2DES [5-7]. Weiss oscillations are a type of $R_{x x}$ oscillations observed in high mobility 2DES with a lateral periodic modulation (superlattice) imposed in one ' direction. In this scenario electrons are subjected simultaneously to two different periodic potentials. One is spacedependent, i.e., the superlattice, and the other is timedependent, i.e., MW radiation. Thus, interesting properties are expected to turn up due to the joint effect of both potentials.

The origin of Weiss oscillations is a periodically modulated Fermi energy as a direct consequence of the spatial periodic potential $V(x)$ imposed on the 2DES. The spatially modulated Fermi energy, (see Fig. 1), changes dramatically the scattering conditions between electrons and charged impurities presented in the sample. At certain $B$ the transport will be more dissipative. This corresponds

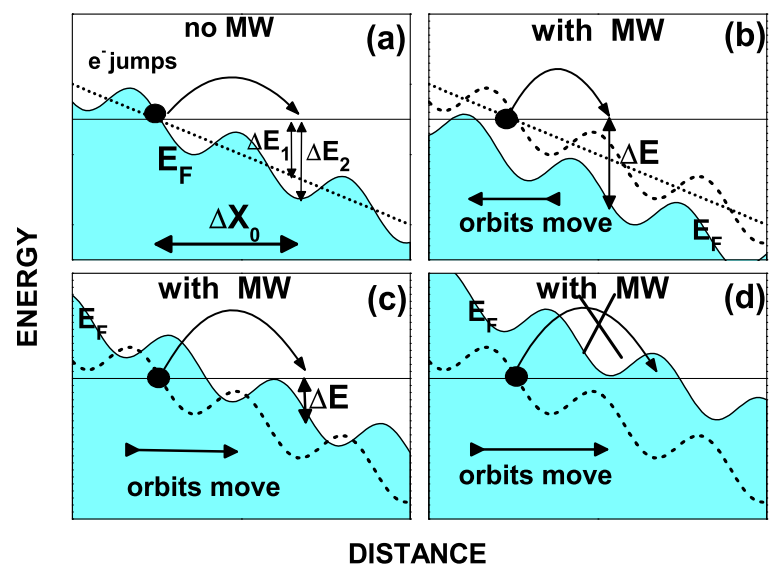

Fig. 1: Schematic diagrams of electronic transport of a 2DES with a spatial periodic modulation in one direction. In Fig.(1.a) no MW field is present. When the MW field is on, the orbits oscillate with $w$. In Fig.(1.b) the orbits move backwards during the jump. In Fig.(1.c) the orbits are moving forwards but still the electronic jump can take place. In Fig. (1.d) the orbits are moving forwards but for a higher MW intensity. Thus the electron movement between orbits cannot take place because the final state is occupied. 
to a peak in $R_{x x}$ [8]. Meanwhile in others, the transport will be less dissipative corresponding to a $R_{x x}$ valley [ 8 . Under this scenario it is expected that the presence of MW radiation will alter dramatically the $R_{x x}$ response of the system. We predict that the combined effect of MW radiation and spatial potential will lead the system to an interference regime with constructive and destructive responses [8]. As a consequence $R_{x x}$ will present a modulated profile. The theory presented here can have potential applications in other fields and systems like nano electromechanical systems (NEMS) 9 with AC-potentials and surfaces acoustic waves (SAW) [10] in 2DES or quantum dots illuminated with MW radiation. In other words, the physics presented in this letter can be of interest for an audience dealing with the effects that $\mathrm{AC}$ or/and DC fields produce on nano-devices.

In this letter we consider at the same footing the effects of both a spatial periodic modulation and MW radiation on the transport properties of a 2DES in the presence of $B$. We propose an alternative approach to study the effects of both potentials based on the fast Fourier transform, (FFT) [11, of the obtained magnetorresistance profiles in function of the inverse of $B$. We first study the FFT of the system for each potential individually. Then we study jointly the FFT of the system when the two types of potentials are acting simultaneously. We obtain two peaks, corresponding to the two harmonic potentials. An interesting scenario arises when the two FFT peaks coincide. Then we can extract information affecting the two potentials. Also it can be of practical interest, for instance a 2DES with a superlattice of a known spatial period can work as a nanosensor to detect radiation of certain frequencies, for instance for the Terahertz band.

Our system consists in a 2DES subjected to a perpendicular $B$ (z-direction) and a DC electric field $\left(E_{d c}\right)(\mathrm{x}-$ direction). We include the unidirectional periodic potential $V(x)=V_{0} \cos K x$ where $K=2 \pi / a, a$ being the spatial period of the superlattice. The total hamiltonian $H$, can be written as:

$$
\begin{aligned}
H= & \frac{P_{x}^{2}}{2 m^{*}}+\frac{1}{2} m^{*} w_{c}^{2}\left(x-X_{0}\right)^{2}-e E_{d c} X_{0}+\frac{1}{2} m^{*} \frac{E_{d c}^{2}}{B^{2}} \\
& +V_{0} \cos (K x)=H_{0}+V_{0} \cos (K x)
\end{aligned}
$$

$X_{0}$ is the center of the orbit for the electron spiral motion: $X_{0}=\frac{\hbar k_{y}}{e B}-\frac{e E_{d c}}{m^{*} w_{c}^{2}}, e$ is the electron charge, $w_{c}$ is the cyclotron frequency. $H_{0}$ is the hamiltonian of a harmonic quantum oscillator and its wave functions, the well-known oscillator functions (Hermite polynomials). We treat $V_{0} \cos (K x)$ in first order perturbation theory and the first order energy correction is given by: $\epsilon_{n}^{(1)}=V_{0} \cos \left(K X_{0}\right) e^{-X / 2} L_{n}(X)=U_{n} \cos \left(K X_{0}\right)$, where $X=\frac{1}{2} l^{2} K^{2}, L_{n}(X)$ is a Laguerre polynomial and $l$ the characteristic magnetic length. Therefore the total energy for the Landau level $n$ is given by: $\epsilon_{n}=\hbar w_{c}\left(n+\frac{1}{2}\right)-$ $e E_{d c} X_{0}+U_{n} \cos \left(K X_{0}\right)$. This result affects dramatically the Fermi energy as a function of distance (see Fig. 1), showing now a periodic and tilted modulation [8].

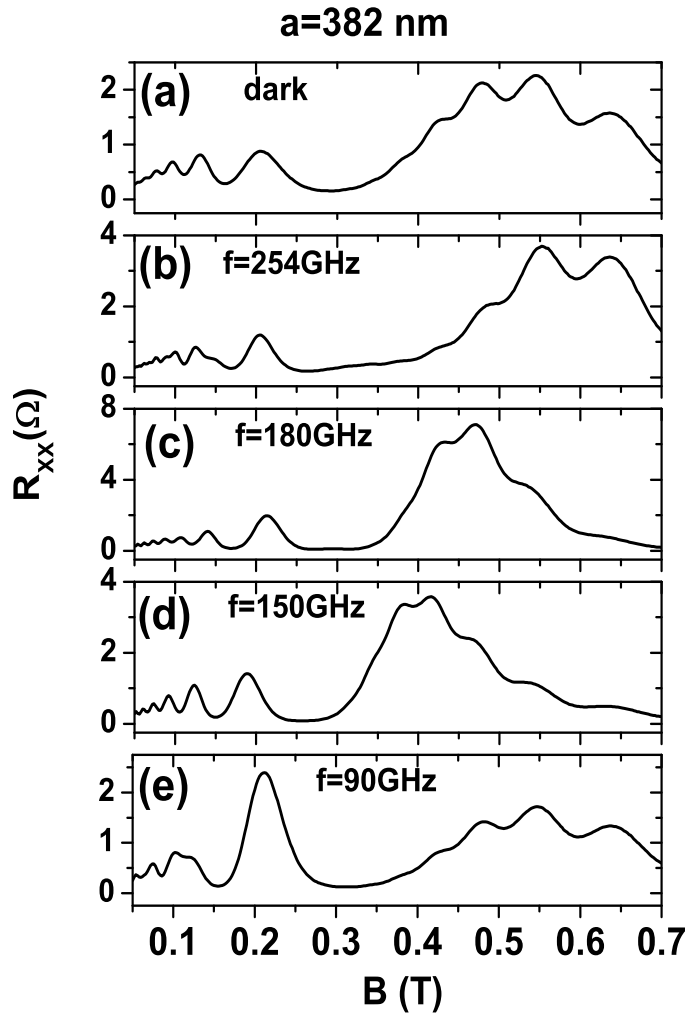

Fig. 2: Calculated $R_{x x}$ as a function of magnetic field $B$ for dark and radiation of different frequencies. The period of the static modulation is $a=382 \mathrm{~nm}$ and the modulation amplitude is $V_{0} \sim 0.1 \mathrm{meV} . \mathrm{T}=1 \mathrm{~K}$.

Now we introduce impurity scattering suffered by the electrons in our model $7,8,12$. When one electron scatters elastically due to charged impurities, its average orbit center position changes, in the electric field direction, from $X_{0}$ to $X_{0}^{\prime}$. Accordingly, the average advanced distance in the $x$ direction is given by $\Delta X_{0}=X_{0}^{\prime}-X_{0} \simeq 2 R_{c}$, $R_{c}=\frac{\sqrt{2 m^{*} E_{F}}}{e B}$ being the orbit radius. There is a direct relation between advanced distance and dissipated energy (see Fig. 1), and when the 2DES is subjected to $V(x)$, the energy variation in the scattering jump has the expression:

$$
\Delta \epsilon \simeq e E_{d c} 2 R_{c}+U_{n}\left[\cos \left(K X_{0}\right)-\cos \left(K X_{0}^{\prime}\right)\right]
$$

After some algebra we can obtain from this expression an averaged energy variation that can be written as 8 :

$$
\Delta \epsilon \simeq e E_{d c} 2 R_{c}-V_{0} e^{-X / 2} J_{0}(2 \sqrt{X}) \cos \left[2\left(R_{c} K+\frac{\pi}{4}\right)\right]
$$

being $J_{0}$ Bessel function of zero order. The advanced distance corresponding to $\Delta \epsilon$ can now be straightforward calculated []:

$$
\Delta X_{T}=2 R_{c}-\frac{V_{0} e^{-X / 2} J_{0}(2 \sqrt{X})}{e E_{d c}} \cos \left[2\left(R_{c} K+\frac{\pi}{4}\right)\right]
$$



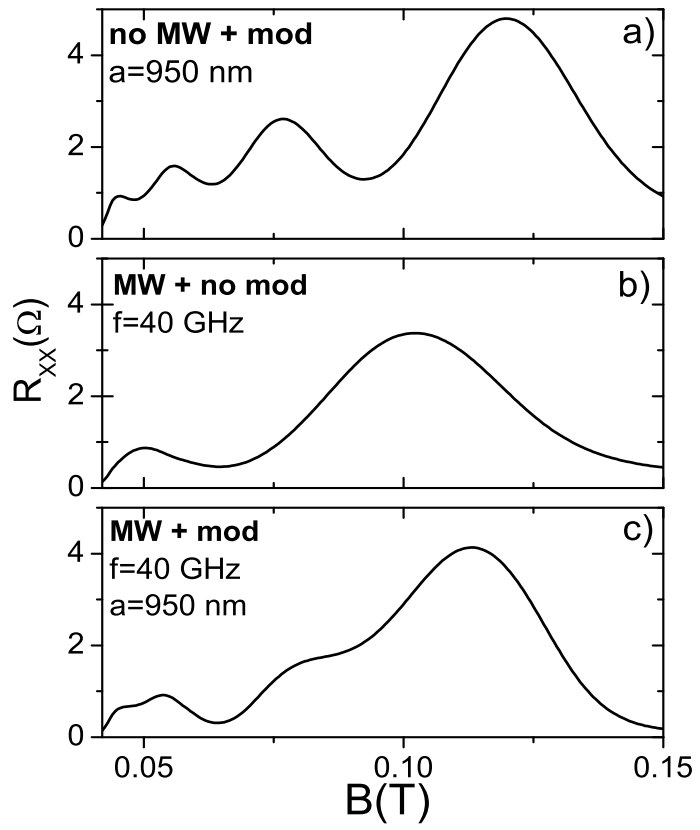

Fig. 3: Calculated $R_{x x}$ vs $B$. In Fig. (a), we plot the case of superlattice with no radiation. Therefore, we represent the Weiss oscillations. Fig. (b) represents the case of a 2DES with no superlattice subjected to radiation. Thus, we obtain the well-know radiation-induced resistance oscillations. In Fig. (c) we represent the joint effect of a one-directional superlattice with radiation. We obtain a modulated $R_{x x}$ profile. The period of the superlattice is $950 \mathrm{~nm}$ and the radiation frequency 40 GHz. $(\mathrm{T}=1 \mathrm{~K}$.

From $\Delta X_{T}$ we calculate a drift velocity for the electron dissipative transport in the $x$ direction and finally we obtain $R_{x x}$ [7,8,12. In Fig. 2a we represent $R_{x x}$ as a function of $B$. The period of the static modulation is $a=382$ $\mathrm{nm}$ and the modulation amplitude is $V_{0} \sim 0.1 \mathrm{meV}$. We reproduce Weiss oscillations with reasonable agreement with experiments [2,3].

If now we switch on the MW field, we expect that its effect will alter dramatically the $R_{x x}$ response of the system. In our simulations we consider that the MW field is plane polarized along the $x$-direction that is the direction of the current and the direction of the spatial potential, i.e., of the superlattice. 11] From the MW driven electron orbits model [7, 8, we propose that MW radiation forces the electron orbits to move back and forth at the frequency of MW. This affects the scattering conditions of the 2 DES increasing or decreasing the distance of the scattering jump giving rise to MW-induced $R_{x x}$ oscillations. Both effects, periodic spatial modulation and MW radiation alter simultaneously the average advanced distance when the electron scatters (see Fig. 1). We obtain an ex-

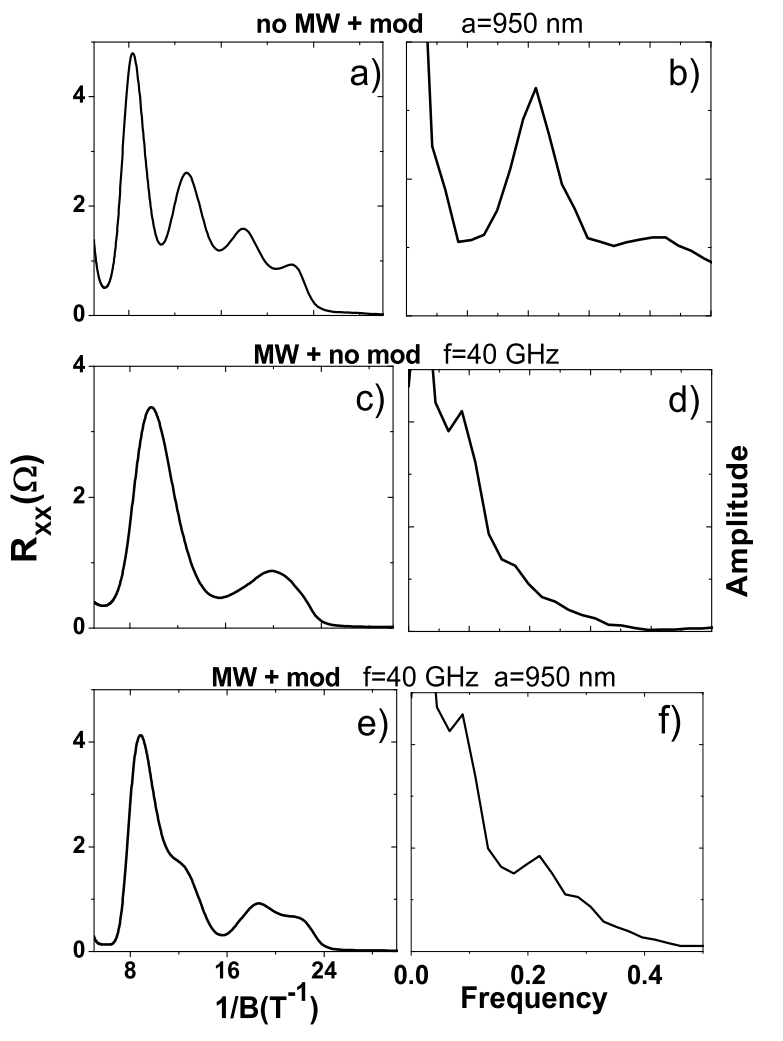

Fig. 4: Left Panels: $R_{x x}$ vs $(1 / B)$. In (a) we represent the case of a superlattice of $\mathrm{a}=950 \mathrm{~nm}$ and no radiation. In (c) we plot the case where the spatial modulation is removed. The frequency of the radiation field is $40 \mathrm{GHz}$. In (e) both spatial and temporal periodic modulations are present. In the right panels we represent the FFT of the corresponding left curves. $(\mathrm{T}=1 \mathrm{~K})$.

pression for the total average distance in the $x$ direction 8]:

$$
\Delta X_{T}=2 R_{c}-S \cos \left[2\left(R_{c} K+\frac{\pi}{4}\right)\right]+A \cos (w \tau)
$$

where $S=\frac{V_{0} e^{-X / 2} J_{0}(2 \sqrt{X})}{e E_{d c}}$ and $A=\frac{e E_{o}}{m^{*} \sqrt{\left(w_{c}^{2}-w^{2}\right)^{2}+\gamma^{4}}} . E_{o}$ is the MW electric field amplitude, $\gamma$ is a phenomenologically introduced [7] damping parameter and $\tau$ the scattering time. From $\Delta X_{T}$ we calculate an electron drift velocity [7, 8, 12] and finally $R_{x x}$ of the system:

$$
R_{x x} \propto-S \cos \left[2\left(R_{c} K+\frac{\pi}{4}\right)\right]+A \cos (w \tau)
$$

According to this expression we can predict an interference regime between both periodic potentials which will be reflected on $R_{x x}$ [8,13] showing a modulated profile when plotted against $B$. We can observe this behaviour in Figs. 


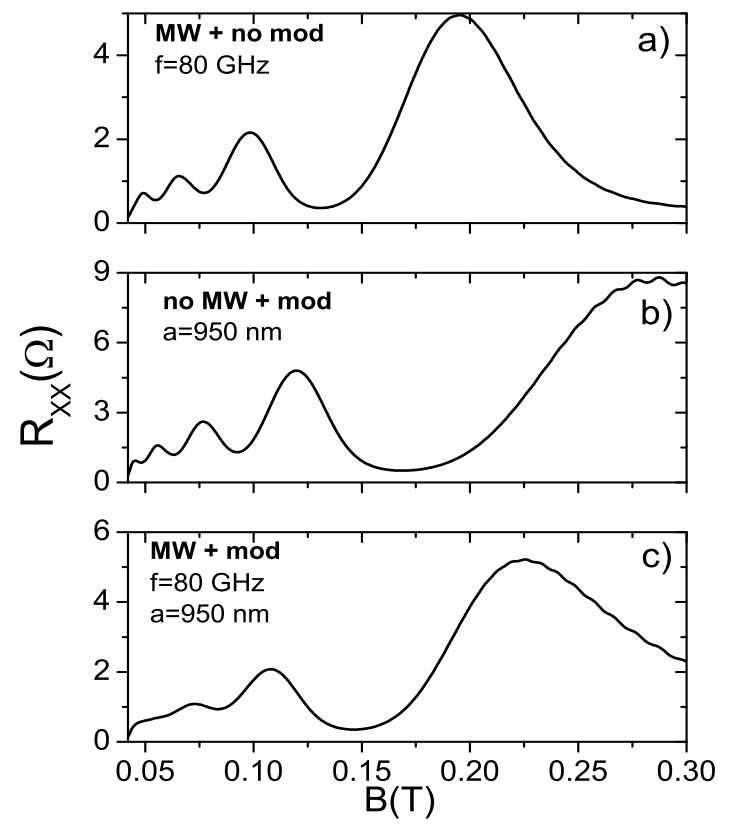

Fig. 5: Calculated $\rho_{x x}$ vs B. Similar panels as in Fig. 3. Now the spatial period of the superlattice is the same but the radiations frequency is $80 \mathrm{GHz} .(\mathrm{T}=1 \mathrm{~K}$.)

2b-2c. In these figures we represent magnetoresistance versus $B$ for a one-directional superlattice $(a=382 \mathrm{~nm})$ with MW radiation of different frequencies: 90, 150, 180 and $254 \mathrm{GHz}$. All of them show different modulated profiles due to the interference effect of both potentials. That means that depending mainly on $a, w$ and for some values of $B, R_{x x}$ will present a constructive response and a reinforced signal. For other values of $B$ the interference will be destructive and the $R_{x x}$ response will be closer to the one of the dark case.

A further approach to analyze this scenario of irradiated Weiss oscillations is based one the FFT of the $R_{x x}$ signal versus the inverse of the applied magnetic field. The goal would be to obtain information from both, superlattice built on the 2DES and radiation. The FFT is carried out on the calculated data of $R_{x x}$ versus $(1 / B)$ because they present harmonic distribution; see left panels of Figs. 4 and 6 where we represent $R_{x x}$ versus the inverse of the magnetic field. However the standard plots of $R_{x x}$ versus $B$ are not harmonic (see Figs. 3 and 5 ). According to equation (6) the spatial part of $R_{x x}$ oscillations depends on the cosine term: $\cos \left[2\left(R_{c} K+\frac{\pi}{4}\right)\right]$, where

$$
2 K R_{c}=\left[2 \frac{2 \pi}{a} \frac{\sqrt{2 m^{*} E_{F}}}{e}\right] \times \frac{1}{B}=k_{e s p} \times \frac{1}{B}
$$

Here the spatial frequency, $k_{e s p}$, is related with the fre-

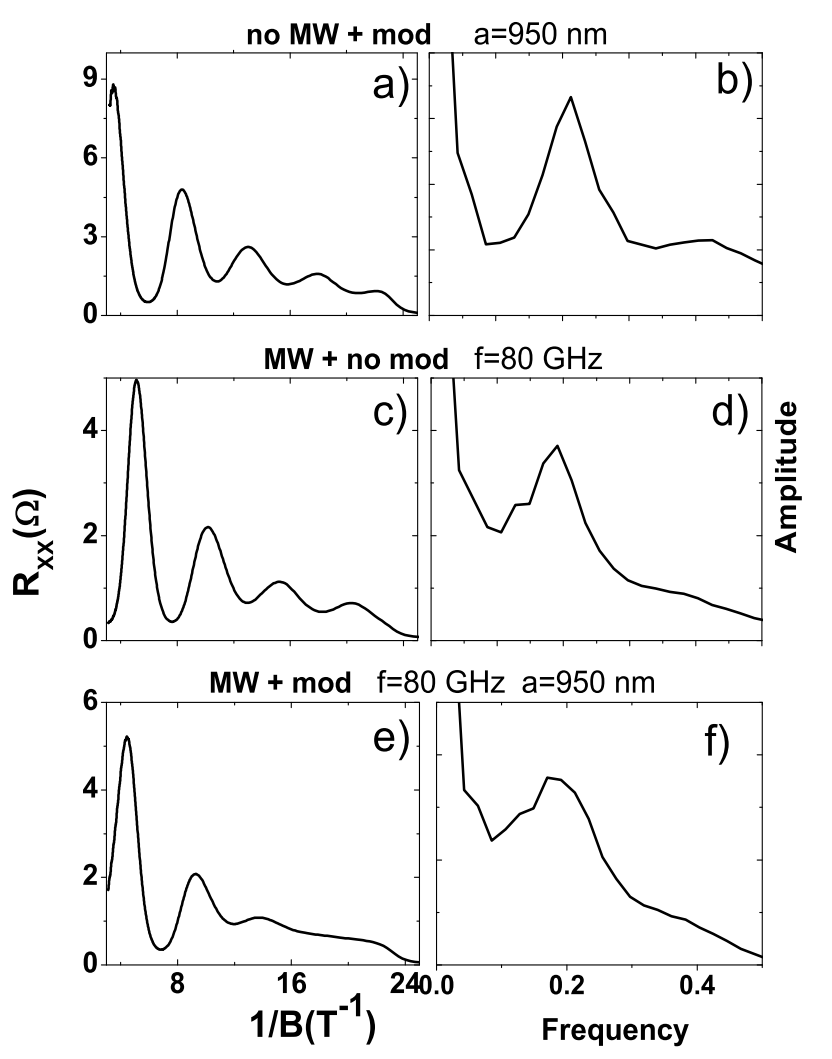

Fig. 6: Calculated $\rho_{x x}$ vs B. Similar panels as in Fig. 4. The spatial period is the same but the radiation frequency is 80 GHz. $(\mathrm{T}=1 \mathrm{~K})$.

quency obtained from the FFT plot, $\nu_{e s p}$ through

$$
k_{e s p}=2 \pi \nu_{e s p}
$$

(see right panels of Fig. 4). From this expression we would be able to obtain information about the spatial period of the superlattice $a$ or even the Fermi level (electron density) if we previously know the frequency from the FFT graph.

We can apply similar procedure for the temporal part of the $R_{x x}$ oscillations. According to equation (6) this part depends on $\cos w \tau$, where $\tau=1 / W$ is the scattering time and $W$ the charged impurity scattering rate which in turn can be expressed as [14:

$$
W=\left(\frac{e^{4} n_{i} m^{*}}{4 \pi \epsilon^{2} \hbar^{3} q_{s}}\right)\left[\frac{1+e^{-\frac{\pi \Gamma}{\hbar w_{c}}}}{1-e^{-\frac{\pi \Gamma}{\hbar w_{c}}}}\right] \simeq\left(\frac{e^{4} n_{i} m^{*}}{4 \pi \epsilon^{2} \hbar^{3} q_{s}}\right)\left[\frac{2 \hbar w_{c}}{\pi \Gamma}\right]
$$

where $n_{i}$ is the impurity density, $\epsilon$ the dielectric constant, $q_{s}$ the inverse of the Thomas-Fermi screening length and $\Gamma$ the Landau level width. Therefore, we can write for $w \tau$, 
similarly as we did for the spatial part:

$$
w \tau=w \times\left(\frac{4 \pi \epsilon^{2} \hbar^{3} q_{s}}{e^{4} n_{i} m^{*}}\right)\left[\frac{\pi \Gamma m^{*}}{2 \hbar e}\right] \times \frac{1}{B}=w_{\text {time }} \times \frac{1}{B}
$$

the angular frequency $w_{\text {time }}$ is related to the frequency of the time dependent FFT $\nu_{\text {time }}$ through

$$
w_{\text {time }}=2 \pi \nu_{\text {time }}
$$

(see right panels of Fig. 6). Thus, knowing $\nu_{\text {time }}$ from the FFT plot, we could obtain information such as the frequency of radiation or material dependent information, for instance scattering time.

In the above approach we have considered individually the potentials acting on the system. A more interesting scenario arises when we study jointly the FFT of the system when acting the two types of potentials simultaneously (Figs. 4f and 6f). We can have two different situations, in the first one the FFT peaks do not coincide, they show up far apart (see top and middle panels of Figs. 4 and 6 ). In this case the obtained information would be as the one described above. On the other hand, when the two FFT peaks coincide, $\nu_{\text {time }}=\nu_{\text {esp }}$ and knowing previous information of one the potentials, either radiation or superlattice, we could obtain in turn important information of the other one. An example of application of the proposed theory it would be the design of radiation sensors; a 2DES with a superlattice of known spatial period could work as a nanosensor to detect radiation of certain radiation frequency, such as the Terahertz band. Using the criteria of coincident peaks, we have calculated that to detect, for instance, radiation of $0.5 \mathrm{THz}$ we would need a superlattice of a spatial period of $a=122 \mathrm{~nm}$.

In conclusion, we have further examined the problem of irradiated Weiss oscillations. We have first obtained the interference and modulated profile of $R_{x x}$ vs $B$ arising from joint effect of superlattice and radiation. Then we have studied this effect from the perspective of the FFT of $R_{x x}$ response vs $1 / B$. We have obtained two peaks either one corresponding to one potential, time or spatial dependent. Two scenarios have been obtained, when the FFT peaks positions are the same and when they do not coincide. The most interesting is the first one, where the design of a nanosensor for Terahertz radiation based on a unidirectional superlattice of known period has been proposed.

This work is supported by the MINECO (Spain) under grant MAT2011-24331 and ITN Grant 234970 (EU).

\section{REFERENCES}

[1] J. Iñarrea and G. Platero Europhys. lett. 40, 417, (1997); J. Inarrea, G. Platero, AH MacDonald, Phys. Stat. Sol. A, 203, 1148, (2006); Jesus Inarrea and Gloria Platero, Nanotechnology, 21, 315401, (2010).

[2] D.Weiss, K. Von Klitzing, K. Ploog and G.Weimann, Europhys. Lett. 8, 179, (1989); R.R. Gerhardsts, D. Weiss and K. von Klitzing, Phys Rev Lett. 62, 1173, (1989); D.Weiss, R.R. Gerhardts, and K. v. Klitzing, Phys. Rev. B, 39, 13020, (1989).

[3] R.W. Winkler, J.P. Kotthaus and K. Ploog, Phys. Rev. Lett., 62, 1177, (1989)

[4] P.Vasilopoulos and F.M.Peeters, Phys. Rev. B, 63, 2120, (1989); F.M.Peeters and P. Vasilopoulos, Phys. Rev. B, 47, 1466, (1993); Phys. Rev. B, 46, 4667, (1992).

[5] R.G. Mani, J.H. Smet, K. von Klitzing, V. Narayanamurti, W.B. Johnson, V. Umansky, Nature 420646 (2002); R. G. Mani, J. H. Smet, K. von Klitzing, V. Narayanamurti, W. B. Johnson, and V. Umansky, Phys. Rev. Lett. 92, 146801 (2004); Phys. Rev. B 69, 193304 (2004); R. G. Mani, Appl. Phys. Lett. 92, 102107 (2008); R. G. Mani, V. Narayanamurti, K. von Klitzing, J. H. Smet, W. B. Johnson, and V. Umansky, Phys. Rev. B, 70, 155310, (2004); R. G. Mani, A. N. Ramanayaka, and W. Wegscheider, Phys. Rev. B, 84, 085308, (2011); R. G. Mani, A. N. Ramanayaka, Tianyu Ye, M. S. Heimbeck, H. O. Everitt, and W. Wegscheider, Phys. Rev. B, 87, 245308, (2013.)

[6] M.A.Zudov, R.R. Lu, N. Pfeiffer, K.W. West, Phys. Rev. Lett. 90046807 (2003)

[7] J. Iñarrea and G. Platero, Phys. Rev. Lett. 94016806, (2005); J. Iñarrea and G. Platero, Phys. Rev. B 72193414 (2005); J. Iñarrea and G. Platero, Appl. Phys. Lett. 89, 052109, (2006); Jesus Inarrea, R.G. Mani and W. Wegscheider, Phys. Rev. B, 82, 205321, (2010)

[8] J. Inarrea and G Platero, Appl. Phys. Lett. 93, 062104, (2008); J. Iñarrea, Appl. Phys. Lett. 90, 172118, (2007); J. Iñarrea, Appl. Phys. Lett. 90, 262101, (2007); A.N. Ramanayaka, R.G. Mani, J. Inarrea and W. Wegscheider Phys. Rev. B, 85, 205315, (2012)

[9] F. Pistolesi and R. Fazio, Phys. Rev. Lett., 94, 036806, (2005).

[10] John P. Robinson, Malcom P. Kennett, Nigel R. Cooper and Vladimir I. Fal'ko, Phys. Rev. Lett., 93, 036804, (2004).

[11] Anton Vogl, Phd Thesis Dissertation, Regensburg University, (2011)

[12] B.K. Ridley. Quantum Processes in Semiconductors, 4th ed. Oxford University Press, (1993).

[13] J. Iñarrea and G. Platero, Appl. Phys. Lett. 89, 172114, (2006); J. Iñarrea, Appl. Phys. Lett. 100, 242103, (2012).

[14] J. Iñarrea, Europhys. Lett. 106, 47005, (2014); J Inarrea and G Platero, Phys. Rev. Lett. 111, 220903, (2013). 